\title{
Linx
}

Linx Revue des linguistes de l'université Paris X Nanterre

59 | 2008

Les conjonctions en diachronie : parcours sémantiques

\section{Réflexions sur l'évolution des conjonctions temporelles en français : le cas de premier que}

Hava Bat-Zeev Shyldkrot

\section{CpenEdition}

Journals

Édition électronique

URL : http://journals.openedition.org/linx/622

DOI : $10.4000 / \operatorname{lin} x .622$

ISSN : 2118-9692

Éditeur

Presses universitaires de Paris Nanterre

Édition imprimée

Date de publication : 1 décembre 2008

Pagination : $33-46$

ISSN : 0246-8743

Référence électronique

Hava Bat-Zeev Shyldkrot, «Réflexions sur l'évolution des conjonctions temporelles en français : le cas de premier que », Linx [En ligne], 59 | 2008, mis en ligne le 01 janvier 2012, consulté le 01 mai 2019. URL : http://journals.openedition.org/linx/622 ; DOI : 10.4000/linx.622 


\title{
Réflexions sur l'évolution des conjonctions temporelles en français : le cas de premier que
}

\author{
Hava Bat-Zeev Shyldkrot \\ Université de Tel Aviv
}

\section{Introduction : concepts généraux}

Depuis la fin des années 1970, avec la renaissance des études de la linguistique historique et la réintroduction du concept de grammaticalisation, la préservation de la dichotomie synchronie-diachronie en tant qu'outil de recherche est fréquemment contestée et il est suggéré ( $c f$. Brugman 1988) qu'une étude synchronique peut éclairer des aspects diachroniques, tout comme une approche diachronique est nécessaire pour la compréhension des faits synchroniques. Leur intégration commune dans une explication grammaticale est considérée comme une méthode légitime pour l'étude du langage humain. Certains linguistes déclarent, en effet, qu'il est impossible de comprendre le fonctionnement des éléments grammaticaux sans avoir examiné au préalable les processus diachroniques auxquels ils ont été soumis (Lehmann 1985, Traugott et Heine 1991, Lichtenberk 1991 inter alia).

Qui plus est, les frontières entre les concepts dichotomiques de lexicalisation et de grammaticalisation, ne semblent pas rigoureuses et résultent probablement du fait que les catégories grammaticales ne sont pas univoques, non plus. Ainsi, la distinction entre les processus de lexicalisation et ceux de grammaticalisation, qui a été admise depuis les travaux de Ch. Lehmann $(1982 / 1985 / 1995)$ il y a une trentaine d'années

\footnotetext{
* Cet article constitue une version remaniée d'une communication présentée au colloque DIACROIV à Madrid en 2008.
} 
environ, est considérée actuellement comme problématique (Giacalone-Ramat \& Hopper 1998, Vincent 1995, Haspelmath 1999 inter alia). Nous avons signalé en (2005) que «les processus agissant sur les unités lexicales pour les rendre grammaticales peuvent être les mêmes que ceux qui entraînent un changement de sens. Toutefois, l'évolution d'un verbe «plein » vers un verbe auxiliaire se distingue de l'affaiblissement ou de l'abstraction d'un substantif, en ce que le premier est considéré comme un processus grammatical et le second comme un processus de nature lexicale ». Il en ressort que c'est le point d'arrivée et non pas le point de départ qui détermine la nature du processus. Prévost et Fagard suggèrent également 2007 :4) que « selon la perspective adoptée, lexicalisation et grammaticalisation peuvent être conçues comme des processus opposés ou non, au moins en ce qui concerne la forme «cible » résultant du processus grammatical ou lexical. ${ }^{1}$ » Par ailleurs, les recherches sur l'évolution linguistique révèlent communément une systématique beaucoup plus suivie dans les changements d'ordre syntaxique et probablement phonologique, que dans ceux de nature sémantique ou lexicale. En effet, si, dans l'analyse des changements syntaxiques, un fonctionnement plutôt régulier s'observe et on se contente alors d'expliquer les cas limites ou les exceptions, dans l'analyse sémantique ou lexicale en revanche, on distingue un certain nombre de tendances générales qui pourraient éventuellement se manifester mais que l'on ne découvrirait qu'a posteriori (Traugott 1990, Marcello-Nizia 2006).

Les tentatives fréquentes d'explication de ce phénomène ont toujours considéré les unités grammaticales comme remplissant un rôle important dans la langue et les changements qu'elles subissent comme influant sur la constitution du système linguistique interne. Inversement, les changements subis par les unités sémanticolexicales sont perçus comme ne modifiant guère ce système. Les descriptions de ces changements sont généralement tenues pour peu rigoureuses et aléatoires.

Cependant, depuis une trentaine d'années, un certain nombre de travaux traçant les parcours d'évolution systématique des unités sémantiques ont été publiés ( $c f$. Sweetser (1990), Traugott et Dasher (2002) inter alia). Ces recherches soulèvent des discussions importantes dont la source serait vraisemblablement l'impossibilité d'établir une distinction catégorielle univoque entre les deux types d'unités.

Or, la classe des conjonctions temporelles suscite chez les linguistes un intérêt particulier tant par le nombre relativement grand de synonymes qui s'y trouvent jusqu'au $17^{\mathrm{e}}$ siècle que par le fait que l'on peut discerner des types distincts de développements. En guise d'exemple, on analysera l'évolution de la locution conjonctive de sens temporel premier que, qui permet d'exprimer la relation d'antériorité des faits dénotés par une proposition par rapport aux faits dénotés par une autre proposition (Bat-Zeev Shyldkrot et Kemmer 1988; Bertin 2001, 2008, Combettes 2008, Verjans 2009). Premier que fait partie d'une série de conjonctions exprimant l'antériorité telles que auparavant que, devant que, primes que qui ont toutes disparu de la langue autour du dix-septième siècle.

\footnotetext{
${ }^{1}$ Voir à ce sujet Brinton et Traugott (2005), Marchello-Nizia (2006).
} 


\section{Etat des lieux}

Plusieurs faits semblent être admis à présent en ce qui concerne les propositions subordonnées circonstancielles (Haiman 1974 ; Harris 1986 ; König 1985 ; ThompsonLongacre 1985 ; Kortmann 1997 Piot 1988). En premier lieu, les critères syntaxiques qui permettent de distinguer une proposition subordonnée d'une principale et même parfois d'une indépendante ne sont pas toujours évidents, contrairement à ce que les grammaires traditionnelles avaient laissé entendre. En second lieu, depuis les travaux de König (1985), et de Kortmann (1997), plusieurs linguistes (Harris 1986 ; König 1985 inter alia) sont de plus en plus conscients du fait que les étiquettes que portent les subordonnées et les locutions conjonctives qui les introduisent, telles que temporelles, causales ou concessives sont fort polysémiques et prêtent à confusion. D'ailleurs, la terminologie qui régit ces locutions n'est pas, non plus, univoque et témoigne des hésitations accompagnant ces marqueurs. En effet, parfois le même terme est intitulé 'conjonction' parfois 'locution conjonctive' et parfois 'subordonnant'. Ces étiquettes pourraient suggérer que les catégories sont des catégories discrètes et qu'il est généralement possible de les distinguer les unes des autres sans trop de difficultés. Or, dans de nombreux cas, il n'est pas possible d'assigner à une locution conjonctive un sens précis, vu que ces catégories sémantiques conceptuelles se fondent l'une dans l'autre, de sorte que le rapport qui existe entre la principale et la subordonnée est souvent ambigu. Notons toutefois que la conjonction n'est pas le seul facteur qui détermine le type de rapport existant entre la subordonnée et la principale, car sa suppression démontre bien que dans beaucoup de cas l'ambiguïté ou l'impossibilité d'assigner une valeur univoque à la proposition demeure. D'autres facteurs qui favorisent ce flottement sémantique entrent probablement en jeu. Il semble cependant que, le plus souvent, c'est la locution qui détermine en dernière analyse le sens de la proposition. Pour ne citer que quelques exemples : selon les cas, quand peut introduire le rapport temporel de simultanéité ou de concomitance, un rapport temporel de postériorité, un rapport de cause et un rapport de concession ou d'opposition (voir par exemple Borlé 1927, Sandfeld 1965, Harris 1986).

1) Tout change, naturellement, quand la paix s'établit dans la région. (simultanéité)

2) Tout ceci compte vraiment quand il s'agit d'expliquer à l'enfant. (simultanéité)

3) Quant il ot chevauchié grant piece, s'entra en une grant forest. (Perlesvaus) [Quand il eut chevauché un grand moment, il entra dans une grande forêt] (postériorité)

4) Or irez vos certes, quant jol cumant. (Roland)

[Vous partirez assurément, puisque je l'ordonne] (cause) 
5) Quand tous les autres avaient pris peur et lâché pied, lui seul s'entêtait à ne pas rentrer à Paris. (A. Daudet) (concession)

6) Quand je voyagerais dans des pays lointains jamais je n'oublierais mon pays natal. (concession).

De même, la conjonction pendant que, par exemple, a introduit aussi bien le temps, la cause ou l'opposition :

7) Et en peut advenir inconveniens innumerables, car les delinquens ce pendant que on yra a juge se absenteront, et ainsi justice sera illudee. (Juvenal des Ursins) (temps-simultanéité)

8) On doit tousjours lessier le plus fort derrière pour resister aux survenans et aux empechemens soudains, pendant que les autres se mettroient en arroy, comme firent ceulx de Crathor. (Jean de Bueil) (opposition)

9) Ce temps pendant que ledict duc mist à venir, quelquung de ceulx du dedans se advisa et apporta des fagotz alluméz pour gecter au visaige de ceulx qui se efforçoient de rompre la porte (Commynes) (tempssimultanéité)

Et même en français contemporain, son emploi est susceptible de traduire plusieurs sens, certains toutefois plus fréquents que d'autres :

10) Pendant que je regardais à la fenêtre, je vis passer un vol d'hirondelles. (temporel)

11) Pendant que vous y étiez, vous auriez dû ramener vos affaires. (causal)

12) Je ne crus néanmoins avoir rien gagné, pendant que je n’entendis que leurs acclamations. (opposition).

Par ailleurs, comme il a été signalé, les classements traditionnels des subordonnées et des subordonnants sont de plus en plus remis en question (Bertin 2008). La distinction entre les différents types de subordonnées adversatives comportant les propositions causales, conditionnelles, concessives et oppositionnelles n'est plus considérée comme pertinente. Déjà Sandfeld (1965) a insisté sur l'ambiguïté possible de telles phrases. De même, Harris (1986) a déclaré que les propositions dites conditionnelles peuvent s'interpréter tantôt comme des subordonnées causales où le rapport de cause est attesté (parce que), tantôt comme des subordonnées conditionnelles où le rapport de cause est supposé (si), tantôt comme des concessives où le rapport de cause entre la subordonnée et la principale est complètement nié (bien que). Cette ambiguïté peut être décelée depuis le français médiéval : 
13) une est que un monde soit apres un autre par succession de temps, si comme aucuns anciens cuiderent que ce monde eust commencement parce que tout estoit devant en une masse confuse sanz ordre, sanz forme et sanz figure, (Oresme, Nicole).

14) Se j'avoie le sens qu'ot Salemons, si me feroit amours pour fol tenir (Couci)

15) Mes il failli a sa cuidance

Et bien qu'il fust de grant puissance, Quer Trajan, l'emperiere honeste, li fist tantost couper la teste (La vie de Saint Grégoire le Grand, I, 1428).

Même les locutions conjonctives qui se prêtent moins en apparence à ce type d'ambiguïté finissent par modifier leur sens tout en accumulant d'autres significations. Un bon exemple est fourni par les locutions selon que et suivant que. Cette dernière est de formation plus tardive en tant que locution et on en retrouve les premiers emplois à la fin du $16^{\mathrm{e}}$ siècle. Bien que les différences sémantiques de ces deux conjonctions soient assez limitées, elles évoluent quand même et penchent vers l'expression de l'alternative.

16) Quanque on li fait sueffre et endure

Sanz courrouz en fait ny en dit,

Selon que l'ermite $\mathrm{m}$ ' a dit. (Miracle de un paroisian esconmenie)

17) Et quant d' entre eulx serez delivres,

Par ces petites prieurtez

Alez, ou seuffrent de durtez

Assés et moinnes et nonnains,

Et la donnez de voz deux mains,

Selon que bon vous semblera

Et ou verrez que bien sera. (Miracle de Saint Bautheuch)

18) La creature de Dieu raisonnable doit entendre, selon que dit Aristote, que des choses invisibles [...]. (Jean d'Arras)

19) Nous choisirons le restaurant, selon que vous préférez la viande ou le poisson.

20) Suivant qu'on m'aime ou hait, j'aime ou hais à mon tour. (Corneille)

Or, toutes les conjonctions, même celles qui introduisent des notions proches, n'évoluent pas de la même manière et ne suivent pas un parcours identique. Il est donc intéressant de connaitre les facteurs qui déterminent les divers parcours qu'empruntent les conjonctions le long de leur évolution. 


\section{Parcours d'évolution}

Plusieurs conjonctions, dont l'usage semble très fréquent jusqu'au $16^{\mathrm{e}}$ et même 17 e siècle, ont pratiquement disparu de la langue française. Il s'agit notamment des locutions conjonctives exprimant le temps telles que auparavant que, devant que, primes que, premier que, soudain que, ains que, etc. Il en est résulté qu'en l'espace de quelques centaines d'années, l'inventaire des locutions conjonctives a été complètement modifié. Beaucoup de locutions ont disparu, d'autres ont été formées et le processus ne semble pas terminé.

Les tentatives de dévoiler les mécanismes qui régissent l'évolution des termes, aussi bien des lexèmes que des unités grammaticales, se sont souvent fondées sur l'évolution des subordonnants, notamment des locutions conjonctives, et ont permis de tracer plusieurs parcours communément reconnus. Pour ce qui est des aspects syntaxiques, les origines de ces subordonnants sont très diverses et ne se limitent pas à une catégorie grammaticale spécifique :

\section{1) Pendant $>$ pendant que, attendu $>$ attendu que, moment $>d u$ moment que, devant $>$ devant que, bien $>$ bien que.}

La conjonction peut donc avoir pour source une préposition, un participe présent d'un verbe, un participe passé d'un verbe, un substantif ou un adverbe. Ce qui est frappant, c'est que dans presque tous les cas, c'est la conjonction qui a disparu ou qui a changé de sens mais pas le terme source - la préposition, le nom et le participe :

22) Puisque - puis, lorsque - lors, alors que-alors, auparavant que- auparavant, devant que - devant, soudain que - soudain.

Dans l'exemple cité les termes sources conservent, en effet, leur sens d'origine (puis, lors, alors, auparavant, devant, soudain), alors que la conjonction acquiert un autre sens (puisque, lorsque, alors que) ou n'est plus en usage (devant que, soudain que, auparavant que).

Par ailleurs, le degré de cohésion ou de figement qui lie les différents termes de la locution conjonctive n'est pas toujours évident et permet de se demander si la fragilité de cette cohésion n'est pas, dans une certaine mesure, la cause du renouvellement constant de ces locutions. En ce qui concerne leur évolution sémantique, Meillet (1915) a déjà abordé ce sujet dans son article bien connu sur le renouvellement des conjonctions, en essayant de déterminer pourquoi certaines conjonctions subsistent dans la langue tout en conservant leur sens, alors que d'autres élargissent ou changent leur sens, et que d'autres encore disparaissent complètement de la langue. Selon lui, ce sont les conjonctions à sens plus spécifique, ayant plus d'autonomie dans la phrase, qui sont les plus instables. Il anticipe ainsi les principes bien connus de la théorie du prototype, principes qui n'ont été formulés que quelques dizaines d'années plus tard, en admettant que des termes désignant des notions plus générales (prototypiques) peuvent subsister dans la langue même lorsqu'ils ont subi des changements sémantiques importants. En revanche, les termes qui expriment des notions plutôt spécifiques tendent donc à disparaître assez régulièrement. Il signale en outre la stabilité des conjonctions latines, qui restent presque intactes, 
contrairement aux locutions conjonctives formées en français qui évoluent, tout en changeant de sens et en suivant un certain parcours. Ceci pourrait, en effet, expliquer pourquoi certaines conjonctions telles que quand et si subsistent dans la langue depuis le latin alors que ja soit que, auparavant que, ains que, de formation française ont disparu. Parmi les parcours plus fréquemment discutés signalons:

23) Spatial > temporel > causal > concessif (Traugott et König 1991) - puisque, since, while.

Temporel >oppositionnel >concessif (Harris 1986) - alors que, tandis que

Temporel $>$ logique (Soutet 1990, Kortmann 1997) - alors que

Conditionnel > concessif conditionnel >concessif (Kortmann 1997) - quand, quand même, quand bien même.

Qualitatif > quantitatif > concessif (Bat-Zeev Shyldkrot 1995) - pour peu que, à moins que, qui plus est.

Modalité $>$ temps $>$ logique (Kortmann, 1997, Bertin 2008)

Ainsi on s'aperçoit que les subordonnants temporels suivent différents parcours. Un subordonnant temporel peut devenir un subordonnant concessif ou causal ou un subordonnant logique. Certains types de subordonnants temporels peuvent même complètement disparaître de la langue.

\section{Parcours d'évolution dans la temporalité : primes que et premier que}

Au premier niveau d'analyse, on pourra marquer un rapport d'antériorité d'une proposition $(\mathrm{X})$ par rapport à une autre $(\mathrm{Y})$, un rapport de simultanéité entre $(\mathrm{X})$ et $(\mathrm{Y})$ ou un rapport de postériorité d'une proposition $(\mathrm{X})$ par rapport à une autre $(\mathrm{Y})^{2}$. Au second niveau, il est possible de déceler pour chaque type de rapport des situations ou des scènes temporelles différentes. Par exemple, la postériorité peut également désigner l'immédiateté ou la durée, la successivité ou la répétition. La simultanéité peut en même temps exprimer la durée ou la continuité.

Or, l'abondance de locutions conjonctives exprimant l'antériorité temporelle a déjà été remarquée par Borlé (1927), Imbs (1956) ainsi que par Haase (1969) et Lerch (1925-34) inter alia. Elles peuvent coexister pendant une certaine période et s'emploient côte à côte très souvent chez le même auteur. Toutes disparaissent progressivement pour laisser la place à 'avant que', qui est devenue la seule conjonction à indiquer cette antériorité. Dans cette perspective, on aimerait examiner l'emploi et le parcours de l'une de ces conjonctions qui se trouve en concurrence avec 'avant que' pour marquer l'antériorité, et qui apparait à la fois sous la forme de primes que et de premier(s) que.

Les conjonctions premier que et primes que signifiant 'avant que' sont formées en synchronie sur premier et primes qui, eux, dérivent du latin primarius ('qui est au premier rang') de primus 'premier', employé comme adverbe. L'évolution de premier 'premièrement'

\footnotetext{
${ }^{2}$ Voir à ce sujet par exemple Herman (1963), Klare (1958) et Piot (1988) qui proposent chacun différents critères pour la classification des conjonctions temporelles.
} 
en premier que 'avant que' représente le développement d'un adverbe ou d'un adjectif ordinal en une conjonction temporelle exprimant l'antériorité. En d'autres termes, l'adverbe ou l'adjectif dont la fonction est d'indiquer la priorité d'un objet ou d'un événement dans une série énumérative entre dans une structure conjonctive qui désigne l'antériorité temporelle. Cette évolution se produit de façon graduelle et on retrouve fréquemment, en particulier dans les premiers emplois, des cas limites où ni l'interprétation ni la fonction ne sont univoques. L'antériorité peut impliquer soit deux propositions, l'une antérieure à l'autre (cf. Pierre est sorti avant que Marie ne soit rentrée), il s'agit alors d'une conjonction de subordination, soit une antériorité ponctuelle signifiant 'avant' par rapport à un certain point de repère ( $c f$. Il faut rentrer avant sept heures). Le dénominateur sémantique commun qui semble avoir permis ce développement est au départ l'idée de primauté ou de priorité et ensuite celle d'antériorité. Il est clair que ces notions sont sémantiquement et conceptuellement liées, le premier désignant celui qui est à la tête, devançant les autres qui le suivent, réellement ou métaphoriquement. Cependant, il est également possible de considérer le rapport d'antériorité exprimé par premier que comme un rapport de comparaison que l'on pourrait qualifier de "préférentielle », entre le constituant antérieur et les suivants, ce qui permettrait de reconnaittre l'origine de la conjonction. On s'aperçoit toutefois que l'idée d'énumération n'est plus toujours présente dans le second cas. Il ne s'agit plus alors d'énumérer des objets ou des événements mais simplement de placer deux propositions dans une séquence temporelle l'une par rapport à l'autre. L'adverbe premier n'existe plus sous cette forme mais a pris la terminaison morphologique adverbiale -ment. La conjonction premier que tout comme devant que, a été remplacée par avant que. Imbs :

Le premier emploi que l'on trouve de cette construction conjonctive, est selon

\section{4) Deis primes saveir son corage}

que t'i embates senz conduit (Thèbes, 1210)

Cette construction conjonctive n'est pas encore figée et l'insertion de plusieurs termes entre le premier élément et le second est possible. Alors que selon Lerch (1925$34)$, cette conjonction ne se rencontre pas avant Froissart, Imbs signale à part $(24)^{3}$, un exemple additionnel qui date de 1363 dans le Voir-Dit de Guillaume de Machaut:

25) Il est raison que le sachiés premier que li autre,

où premier que correspond à 'avant'. On pourrait suggérer que li autre joue le rôle d'une proposition elliptique qui équivaut à premier que li autre le sache $e^{4}$.

Dans les exemples plus tardifs, les deux termes tendent plutôt à apparaitre de manière contiguë, bien qu'on rencontre toujours des occurrences où ils sont disjoints l'un de l'autre.

\footnotetext{
${ }^{3}$ Il existe d'ailleurs une variante premiers... que, qui remplace dans un manuscrit daté de 1288 primes que.

${ }^{4}$ Les emplois elliptiques se rencontrent même beaucoup plus tard : «ceulx-là entrèrent premier que nous » (Commynes, Mémoires, T.1, 1489-1491, 161).
} 
26) Pense, pense, premier que tu diez rien, quant es et quant grant sont esté plus grans que toy sans comparoison - degeté et debouté de ceste grace quy t'est donnee, et que a celle ne sont peu parvenir. (Pierre Crapillet, Cur Deus homo; De arrha animae, c.1450-1460, 278)

27) Or entendez bien, vous toutes qui cy estes presentes, je vous avertis que jamais on ne doit tirer espee nue ne autre long trenchant devant femme grosse, que premier que riens s'en face, ne lui va doucement touchier du plat sur son chief affin qu'elle demeure asseuree et que son fruit en soit toute sa vie plus hardy. (Anonyme - Les Evangiles des quenouilles 1, c.1466-1474, 86)

28) Je m'y assayeré, Mes il fault premier que tramecte

Chasser pour avoir viande necte. (La Passion d'Awvergne, 1477, 139)

29) S'ung en avoyes qui fust mon frere, Et j'eusse femme ung peu mignonne, Je lairoye toute la besongne

Premier que m'en puisse garder. (Sottie des rapporteurs, c.1480, 64)

Borlé (1927) mentionne qu'il n'a trouvé que quatre exemples de premier que: deux dans les Discours admirables de Palissy et deux dans l'Heptaméron de Marguerite d'Angoulême. Voici les exemples qu'il cite :

30) $[\ldots]$ aucune fois que j'avais dedens le four diverses couleurs d'esmaux, les uns estoyent bruslez premier que les autres fussent fondus (Discours, 215).

31) $[\ldots]$ le verd des lézards estoit bruslé premier que la couleur des serpens fût fondue... (Discours 215).

32) $[. .$.$] Dieu m'a fait la grace de morir, premier que la violence de mon$ amour ayt mis tache à ma conscience et renommée (Ib., I. 263)

33) Mais bien ay oy que longuement a pesé sur sa bride premier que y voloir conclurre; (Chastellain, Robertet, Montferrant, Les Douze dames de rhétorique, 1462-1463, 134)

Or, la Base de Moyen Français présente plusieurs autres exemples qui témoignent de la fréquence de cette conjonction. Il existe, comme nous l'avons signalé, des cas d'ambiguiité de nature plutôt syntaxique mais également sémantique, où l'emploi de premier que n'est pas univoque, en particulier, lorsqu'il n'est pas suivi d'un verbe.

D'ailleurs, on pourrait se demander s'il s'agit toujours d'une conjonction de subordination dans les cas où il n'est pas suivi de verbe, comme l'on peut voir, dans 
les exemples (34-36). Dans (34), premier que signifie 'avant' mais introduit également l'idée de la primauté d'une ville par rapport aux autres villes et dans (35), premier que

indique la priorité accordée à l'auteur par rapport au roi ; dans (36), il s'agit de la priorité dans l'ordre de succession. L'exemple (37) de Christine de Pisan témoigne plus encore de la fusion sémantique qui était à l'origine de cette locution. D'une part premier que fait ressortir l'importance de l'acte accompli par rapport à la potentialité et d'autre part, il dénote l'antériorité de la potentialité par rapport au fait réalisé.

34) Et, fut à cause que à l'heure du trespas de son père il fist son entrée en Gand premier que en nulle autre ville de son pays, ayant ceste oppinion que c'estoit la ville de son dict pays où il estoit le plus aymé, et que, à l'exemple de ceste là, se rengeroient les autres. (Commynes, Mémoires, t.1, 1489-1491, 118)

35) Et, sur l'heure dont je parle, vint devers ledict seigneur ung gentil homme appellé Loys de Civille, serviteur du connestable et ung sien secretaire nommé Richier, qui tous deux vivent encores, et dirent leur crean et à monsr de Bouchaige et à moy premier que au roy, car le plaisir dudict seigneur estoit tel. (Commynes, Mémoires, t.2, 1489-1491, 49)

36) Et adoncq parla le Jouvencel à sa femme et lui dist en ceste manière: «Madame, je scay pour certain que le Roy vostre pere a ung très beau filz, qui est vostre frere et doit heriter au royaume d'Amydoine premier que vous, par raison. (Jean de Bueil Le Jouvencel, t.2, 1461-1466, 254)

37) Et ainsi il appert que fait, se non en temps, toutesfois quant a nature ou a entencion est premier que puissance. (Christine de Pizan, le Livre de l'advision Cristine, 1405, 74)

On note, par ailleurs, que cette conjonction pouvait apparaitre au $16^{\mathrm{e}}$ et au $17^{\mathrm{e}}$ siècle suivie d'un infinitif avec ou sans la préposition de, évoquant alors uniquement l'antériorité. Soient les exemples :

38) premier que d'avoir mal ils trouvent le remède (Malherbe)

39) Premier que de courir, ces guerriers bien appris, Yront autour du camp (Ronsard, Bocage, cité par Godefroy)

40) Depuis qu'ils sont condamnés des médecins, ils les enterrent, premier que mourir, ceulx qui sont cause de leur mort (Duplessis-Mornay, cité par Borlé 55)

41) Comme en luy nous avons parfaicte fiance et ainsi qu'il eust voullu pour lui estre fait se premier que nous fust decedé. (Jean Le ClercInterpolations et variantes de la Chronique scandaleuse, c.1502, 283) 
Dans l'évolution de premier que, plusieurs aspects méritent d'être évoqués. Le premier concerne le développement d'un item lexical numéral en une conjonction temporelle. Cette évolution n'est pas propre à premier que. D'autres conjonctions suivent le même parcours. Certains chercheurs ont même suggéré que ce rapport est dû à des principes fondamentaux de l'organisation cognitive humaine, qui établissent des concepts comme le temps, sur la base des stratégies de la perception humaine $(\mathrm{H}$. Clark, 1973, Sweetser, 1990). Le second aspect concerne la fonction des conjonctions dans le discours. En effet, si l'on examine la fonction prédominante et caractéristique dans l'acte de communication, l'on se rend compte qu'elle est surtout de nature pragmatique. En réalité, la présence des conjonctions constitue l'un des moyens les plus fréquents permettant la création d'un discours cohésif. En d'autres termes, leur rôle consiste à lier les éléments du discours.

A la lumière de ces faits, il est possible de concevoir le passage du stade adverbial ou prépositionnel au stade conjonctif comme un passage qui se déroule à trois niveaux différents: le niveau syntaxique, le niveau sémantique et le niveau discursif. (antériorité).

Premier $\rightarrow$ premier que (signalant la priorité ou la primauté) $\rightarrow$ premier que

\section{Conclusion}

Cette étude soulève un certain nombre de questions qui méritent d'attirer l'attention des linguistes. Par exemple, quelles sont les catégories sémantiques qui se prêtent plus facilement que d'autres à la grammaticalisation et se renouvellent très fréquemment? Cette question a été abordée à plusieurs reprises par Heine et Kuteva (2002, 2005), qui fournissent une liste des parcours sémantiques. Cependant, on continue à se demander pourquoi le français utilise à un moment donné de l'histoire un si grand nombre de conjonctions exprimant l'antériorité. Ces conjonctions finissent par disparaître et sont remplacées par avant que. On se demande également comment rendre compte du parcours qui fait évaluer le concept de priorité ou de primauté vers celui de l'antériorité (premier que). Enfin, on pourrait s'interroger sur l'origine de cette abondance des conjonctions temporelles, quelle que soit leur nature, par rapport aux autres conjonctions exprimant d'autres concepts, tels que la manière, la cause ou le lieu. 


\section{RÉFÉRENCES BIBLIOGRAPHIQUES}

BAT-ZEEV SHYLDKROT, H., 1995, « Présentation : synchronie et diachronie : du discours à la grammaire », Langue française, 107 , p. 3-6

BAT-ZEeV SHYLDKROT, H., 2005, «Grammaticalisation, changements sémantique et polysémie : le cas de vers et envers », La polysémie, O. Soutet (éd.), PUPS, p. 203-229.

BAT-ZeEV ShyldKrot, H., \& Kemmer S., 1988, «Le développement sémantique des conjonctions en français : quelques concepts généraux », Revue Romane, 23,1, p. $9-20$.

BERTIN, A., 2001, "Maintenant: un cas de grammaticalisation?", Langue française, 130, p. $42-65$.

BERTIN, A., 2008, «La locution ainsi que en Moyen français », Linx, 59, (ce volume).

BORLÉ, E., 1927, Observations sur l'emploi des conjonctions de subordination dans la langue $d u$ XVTe siècle, Paris, Les Belles Lettres.

Brinton, L., \& TraugotT, E.C., 2005, Lexicalisation and Language Change, Cambridge, Cambridge University Press.

Brugman, C., 1988, The story of over: Polysemy, semantics, and the structure of the lexicon, New York, Garland Publishing, Inc.

Clark, H., 1973, "Space, time, semantics and the child », Cognitive Development and the acquisition of language, T. E. Moore (ed.), New York, Academic Press.

Combettes, B., 2008, «L'opposition que / ce que dans les locutions temporelles », Linx, 59, (ce volume).

GiacAlone-RAmat, A., \& Hopper, J. P. (eds.), 1998, The Limits of Grammaticalization, Amsterdam/Philadelphia, John Benjamins.

HAASE, A., 1969, Syntaxe française du XVIIe siècle, Paris, Delagrave, München, M. Hueber.

Haiman, J., 1974, «Concessives, conditionals, and verbs of volition», Foundations of Language 1, p. 341-360.

HARRIS, M., 1986, « Aspects of subordination in English and other languages », Bulletin of the John Rylands University Library of Manchester, 69, 1, p.195-209.

HASPELmath, M., 1999, "Why is grammaticalization irreversible? », Linguistics 37.6, p. $1043-68$.

Heine, B., \& Kuteva, T., 2002, World Lexicon of grammaticalization, Cambridge, Cambridge University Press.

Heine, B., \& KuTEva, T., 2005, Language contact and grammatical change, Cambridge, Cambridge University Press.

HERMAN, J., 1963, La formation du système roman des conjonctions de subordination, Berlin, Akademieverlag. 
IMBS, P., 1956, Les propositions temporelles en ancien français. La détermination du moment, Paris, Les belles lettres.

KLARE, J., 1958, Entstehung und Entwicklung der konz̧essiven Konjunktionen im Französischen, Berlin, Akademieverlag.

KÖNIG, E., 1985, «On the history of concessive connectives in English. Diachronic and synchronic evidence », Lingua, 66, p. 1-19.

Kortmann, B., 1997, Adverbial Subordination. A Typology and History of Adverbial subordinators Based on European Languages, Berlin-New York, Mouton de Gruyter.

LEHMANN, C., 1985, "Grammaticalization : Synchronic Variation and Diachronic Change », Lingua e Stile, XX/3, p. 303-318.

LEHMANN, C., 1982/1985/1995, Thoughts on grammaticalization, Munich, Lincom-Europa.

LERCH, E.,1925-1934, Historische fran₹ösische Syntax, Tübingen, Niemeyer.

LiCHTENBERK, F., 1991, "On the gradualness of grammaticalization », in Traugott, E., \& Heine B., (eds.) Approaches to Grammaticalization, Amsterdam, Benjamins, p. 37-80.

MARCHELlO-NizIA, Ch., 2001, « Grammaticalisation et évolution des systèmes grammaticaux », Langue française, 135, p. 33-41.

MARCHELlo-NizIA, Ch., 2006, Grammaticalisation et changement linguistique, Bruxelles, de boeck.

MeILlET, A., 1915, «Le renouvellement des conjonctions », Annuaire de l'Ecole pratique des Hautes Etudes [Réimprimé in Linguistique historique et linguistique générale, Paris, Champion, 1958].

PIOT, M., 1988, «Coordination-subordination : Une définition générale », Langue française, 77, p. 5-19.

Prevost, S., \& FAGARD, B., 2007, «Présentation: Grammaticalisation et lexicalisation : la formation d'expressions complexes », Langue française, 156, p. 3-9.

SANFELD, Kr., 1965, Syntaxe du français contemporain. Les propositions subordonnées, Genève, Droz.

SOUTET, O., 1990, La concession en français des origines au XVTe siècle, Genève, Droz.

SOUTET, O., 1992, La concession dans la phrase complexe en français, Genève, Droz.

SOUTET, O., 2008, "Genèse des locutions conjonctives concessives du français ", Linx, 59 (ce volume).

Sweetser, E. E., 1990, From Etymology to Pragmatics: Metaphorical and Cultural Aspects of Semantic Structure, Cambridge, Cambridge University Press.

TAlmy, L., 1983, « How language structures space », in Pick, H. \& L. Acredolo (eds.) Spatial orientation: Theory research and application, New York, Plenum Press, p. 225282. 
Thompson, S., \& Longacre, R., 1985, «Adverbial Clauses » in Shopen, T. (ed.) Language Typology and Syntactic Description, Vol. II, Complex Constructions, Cambridge, Cambridge University Press.

TRAUGOTT, E.C., 1990, «From less to more situated in language : the unidirectionality of semantic change ", in Adamson, S., et al. (eds.) Papers from the 5 th International Conference on English Historical Linguistics, Amsterdam, Benjamins.

Traugott, E.C., \& Heine, B., 1991, Approaches to grammaticalization, Amsterdam, Benjamins.

TraugotT, E.C., \& KÖNIG, E., 1991, "The semantics-Pragmatics of Grammaticalization revisited », in Traugott, E.C., \& König, E. (eds.), p. 189-218.

TraugotT, E.C., \& Dasher, R. B., 2002, Regularity in Semantic Change, Cambridge, Cambridge University Press.

VERJANS, T., 2009, Essai de systématique diachronique : Genèse des conjonctions dans l'histoire du français (IX'e-XVII siècles), Thèse de Doctorat, Paris-Sorbonne.

VINCENT, N., 1995, «Exaptation and grammaticalization ». In Andersen H. (ed.) Historical Linguistics, Amsterdam/Philadelphia, John Benjamins, p. 433-445.

\section{Bases de données}

BFM : Base de Français Médiéval, [en ligne]. Lyon: UMR ICAR/ENS-LSH, 2006, $<$ http://bfm.ens-lsh.fr $>$.

DMF : Dictionnaire du Moyen Français. ATILF / Nancy Université - CNRS. Site internet: http://www.atilf.fr/dmf. 